\title{
Fuzzy Based Dynamic Buffer Management in Wimax 16m Networks
}

\author{
${ }^{1}$ Karunkuzhali, D. and ${ }^{2}$ Dinesh Chandra Tomar \\ ${ }^{1}$ Department of CSE, Sathyabama University, Chennai, India \\ ${ }^{2}$ Department of CSE, \\ Shree Motilal Kanhaiyalal Fomra Institute of Technology, Chennai, India
}

Received 2012-07-03, Revised 2012-11-04; Accepted 2012-11-08

\begin{abstract}
In WiMAX 16m networks, buffer allocation is the major problem to be handled for offering Quality of Service (QoS). The lack of buffers increases the packet loss and queuing delay. In this study we propose a fuzzy based dynamic buffer management in WiMAX 16 m network. The Base Station (BS) estimates the parameters such as number of user requests, flow rate, queue length and received signal strength for each user and updates them periodically. When a request arrives at BS, buffer allocation factor is estimated by applying fuzzy logic over these parameters. Then the flow request with high buffer allocation factor is admitted first and rest of the flow requests waits in a queue. Upon new request arrival, if its buffer allocation factor is low, the request is rejected. Otherwise, the pending request packet in the queue is emptied on analyzing their channel condition and buffer is allocated for new request. By simulation results, we show that the proposed technique reduces packet losses.
\end{abstract}

Keywords: Buffer Allocation Factor (BAF), Base Station (BS), Quality of Service (QoS), Subscriber Station (SS), Base Station (BS)

\section{INTRODUCTION}

\subsection{Worldwide Interoperability for Microwave Access (WiMAX)}

An evolving technology for aiding the broadband wireless access in metropolitan area is IEEE 802.16 which is otherwise denoted as Worldwide Interoperability for Microwave Access (WiMAX) (Jin, 2010). This standard offers high range and speed in contrast to IEEE 802.11 (otherwise called as Wireless Local Area Networks, WLAN) WiMAX includes Subscriber Station (SS) and Base Station (BS). BS is linked to core network and is in charge for communication among the SS and core network (Riizwan and Ibrahiim, 2011).

Access Service Network (ASN) manages the communications among the SSs and BS. WiMAX also utilizes the function offered by Connectivity Service Network (CSN) for linking SSs. This network permits any two SSs to communicate directly when they are within transmission range of one another. Or else, another intermediate node is required to connect $\mathrm{SS}$ or BS (Mardini and Alfoul, 2011).
The two models exist in WiMAX which are described below:

- Point to multipoint (PMP) networks: An access network with small number of SSs linked to a full functional BS is referred to as PMP networks

- Multipoint to Multipoint network: A network devoid of centralized BS and each SS possessing capacity to link directly to another SS or through intermediate SSs. This network is otherwise called as mesh network (Mardini and Alfoul, 2011)

The application of WiMAX includes supporting long transmission range and high data rate compared to cellular and WiFi network (Mardini and Alfoul, 2011).

\subsection{Characteristics of WiMAX}

The characteristics of WiMAX networks are as follows:

- It utilizes Orthogonal Frequency Division Multiple Access (OFDMA)

- $\quad$ Spectrum width varies from $1.25 \mathrm{MHz}$ to $28 \mathrm{MHz}$

Corresponding Author: Karunkuzhali, D., Department of CSE, Sathyabama University, Chennai, India 
- Time and Frequency Division Duplexing (TDD and FDD)

- Advanced antenna techniques such as beam forming, Multiple Input Multiple Output (MIMO)

- Per subscriber adaptive modulation

- Advanced coding techniques such as space-time coding and turbo coding

- Strong security and

- Multiple QoS classes appropriately designed for voice and also for a combination of data, voice and video services (So-In et al., 2009)

\subsection{WiMAX $16 \mathrm{~m}$}

The IEEE $802.16 \mathrm{~m}$ system is otherwise called the gigabit WiMAX. This system increases the throughput up to $1 \mathrm{Gbps}$. In addition it intends to maintain inherited IEEE 802.16 standards and interworking with other wireless systems that includes 3GPP LTE and IMTadvanced system (Kim et al., 2012). $802.16 \mathrm{~m}$ technology facilitates more efficient and data communications. It operates both in Time Division Duplex (TDD) and Frequency Division duplex (FDD) mode. Its channel bandwidth is focused up to $20 \mathrm{MHz}$ channels and its peak data rate becomes $350 \mathrm{Mbps}$ (Soni and Kaushal, 2011).

Characteristics of WiMAX $16 \mathrm{~m}$ :

- Its aggregate data rate is $100 \mathrm{Mbps}$ for mobile stations and $1 \mathrm{Gbps}$ for fixed networks

- Its Duplexing schemes includes Time division Duplexing (TDD) and Frequency Division Duplexing (FDD)

- The time taken for transition from idle to active state is $50 \mathrm{~ms}$

- The quality of service classes includes Unsolicited Grand Service (UGS), non-real time poling service (nrtPS), extended real time poling service (ertPS), real time poling service (rtPS) and Best Effort (BE)

- It operates in radio frequency less than $6 \mathrm{GHz}$

- The high speed mobility range is $350 \mathrm{~km} \mathrm{~h}^{-1}$ (Papapanagiotou et al., 2009)

\subsection{Buffer Management}

The method by which the buffer space is distributed among the various output queues can be described using buffer management. The factors to be considered while designing a buffer management algorithm are as follows:

- Packet loss ratio: It is defined as the ratio of number of packets dropped to the total number of packets received
- Hardware complexity: The total hardware required to implement a buffer management algorithm (Thavasi and Natarajan, 2011)

Buffer management is a fundamental technology which controls the assignment of buffer resources among different traffic classes and aggregation of the same according to certain policies. An efficient buffer management policy is required to decide at each step which of the messages is to be dropped when buffer is full and which of the messages are to be transmitted when bandwidth is limited irrespective of the routing algorithms used.

The functions that are to be supported by the buffer management scheme are as follows:

- Regulating insensitive flows and fairness

- Low delay and low delay-jitter values

- Smooth sending rates for each flow

- Avoiding overflow and underflow by controlling the queue size

Necessity of Buffer Management:

- Traditionally, the Buffer management is utilized mainly to regulate the traffic fluctuations (Lenas et al., 2011)

- When a node receives more data to forward, the excess data has to be buffered. When the limited buffer space is full, the congestion occurs and consequently the received data has to be dropped

- The main causes for packet loss in networks are buffer overflows due to congestion. In cases where the underlying traffic has inter-packet dependencies, indiscriminately dropping packets upon overflow may result in very poor performance (Scalosub et al., 2010)

- The performance is high under infinite buffer and infinite bandwidth assumption, however, in real situations, the performance is worse when the buffer and bandwidth are limited due to the congestion control problem

- In order to overcome the above issues, an efficient buffer management scheme is required

\subsection{Problem Identification}

In study (Mardini and Alfoul, 2011) they did not consider mobility set into consideration. Also there is no approach specified for situation that involves the SSs to enter and leave the network on regular basis.

The resource allocation technique described in study (Xue et al., 2007) cannot be directly applied in the 
context of wireless networks due to unique characteristics of the shared wireless channel.

The active queue management proposed in (Lakkakorpi et al., 2007) does not give solutions to packet drops.

Moreover, as per the literature survey, there are only less works related to buffer management in WiMAX.

Hence in this study, we propose a fuzzy based dynamic buffer management in WiMAX 16 m networks.

\subsection{Related Work}

Mardini and Alfoul (2011) have proposed a Modified Weighted Round Robin (MWRR) scheduling algorithm for WiMAX. Their algorithm prevents the issues caused by weighted round robin algorithm WRR that causes unnecessary delay for lower class of services. Their algorithm minimizes the average delay and enhances the average throughput particularly for lower classes by increasing the size of service round in WRR. Their algorithm did not consider the mobility scenario.

Mallapur et al. (2010) proposes a fuzzy based buffer management scheme that performs buffer allocation and packet dropping for wireless multimedia networks in the context of future generation cellular networks. Buffers are allocated to requesting application by using buffer allocation factor and packets are dropped for an application by using dropping factor. A buffer allocation factor for requesting application is computed adaptively based on three fuzzy parameters of an application, namely, priority, rate of flow and packet size.

Thavasi and Natarajan (2011) have proposed an effective queue mangement technique for data communication. They used queues to smooth spikes in incoming packet rates and to allow the router sufficient time for packet processing. When the waiting time is less a better queue management is ensured thereby resulting in increased flow in the queue. When the incoming packet rate was higher than the router's outgoing packet rate, the queue size was increased, eventually exceeding the available buffer space. In their approach, the congestion was detected early and the packets were dropped.

Xue et al. (2007) investigates the problem of TCP performance anomaly issue over multi-rate wireless networks. It presents an optimization-based framework for flow control in multi-rate wireless networks. This framework provides analytical insight to the performance anomaly problem of TCP and establishes a theoretical foundation for a joint flow control and active queue management scheme that solves this problem. The presented active queue management scheme operates based on channel conditions as well as queue lengths.
Lakkakorpi et al. (2007) have studied the end-to-end performance of different applications in WiMAX network with and without Active Queue Management (AQM). Their AQM technique helps in minimizing the queuing delay caused by Base Station Downlink (BSDL) buffering. As their technique offers adequate buffer space, the TCP goodput can be maximized. However, their approach could be applied to Best effort and non real time polling service connections only.

Riizwan and Ibrahiim (2011) have proposed a quality of service architecture for base station. Their approach makes use of the available bandwidth that is left unused or wasted that further leads to efficient use of WiMAX resources. They proposed a combined approach of queue management and bandwidth allocation algorithm for difference services. They did not take the connection blocking and dropping states into consideration for reducing the connection requests.

\section{MATERIALS AND METHODS}

\subsection{Fuzzy Based Dynamic Buffer Management 2.1.1. Overview}

In this proposal, we proposed a fuzzy based dynamic buffer management in WiMAX 16m network which performs buffer allocation and packet dropping. This technique operates in the Base Station (BS). As per application requirements, BS estimates the parameters such as number of user requests, flow rate, queue length and received signal strength and updates periodically. When a buffer request packet arrives, buffer allocation factor (BAF) is estimated using the fuzzy logic applied over the parameters estimated in the BS. The user requests are sorted in the descending order of BAF. This reveals that the flow request with more BAF is admitted and rest of the flow requests await in queue. When a new request arrives, its BAF is tested. If the value is low, the request packet is dropped. Otherwise, the pending request packet in the queue is emptied on analyzing their channel condition and buffer is allocated for new request.

\subsection{Estimation of Metrics \\ 2.2.1. Estimation of Queue Length and Flow Rate}

Let $Q_{i j}(t)$ be the queue length of aggregated traffic flow of service type $j,(j \in[1,2]$ for direct and relay cooperation transmission modes respectively) at base station $\mathrm{i}(\mathrm{i} \in\{1,2, \ldots ., \mathrm{M}\})$ at time $\mathrm{t}$. 
The vector of the queue status of all base stations is given as:

$$
\left.\mathrm{Q}=\left\{\mathrm{Q}_{11}(\mathrm{t}), \mathrm{Q}_{12}(\mathrm{t}) \ldots ., \mathrm{Q}_{\mathrm{M} 2}(\mathrm{t})\right\}\right]^{\mathrm{T}}
$$

By considering the liquid fluid model, the queue length is evaluated using Eq. 1:

$$
\begin{aligned}
& \mathrm{Q}(\mathrm{t})=\mathrm{N}(\mathrm{t}) \operatorname{IR}(\mathrm{t})-(1-\mathrm{R}) \mathrm{BW}_{\mathrm{ij}}(\mathrm{t}) \eta_{\mathrm{ij}} \\
& \forall \mathrm{i} \in\{1,2, \ldots \mathrm{M}\}, \mathrm{j} \in\{1,2\}
\end{aligned}
$$

Where:

$$
\begin{aligned}
& \mathrm{N}_{\mathrm{ij}}(\mathrm{t}) \quad=\text { Number of base stations } \\
& \operatorname{IR}(\mathrm{t})=\text { Input traffic flow to the subscribed base } \\
& \text { station } \\
& \mathrm{N}_{\mathrm{ij}}(\mathrm{t}) \operatorname{IR}(\mathrm{t})=\text { Aggregate downlink flow rate at base } \\
& \text { station i } \\
& \mathrm{R}_{\mathrm{ij}} \quad=\text { average Packet Error Rate (PER) for } \\
& \text { abstracting the channel quality } \\
& \eta_{\mathrm{ij}}=\text { Average spectral efficiency (in bits/s/Hz) } \\
& \mathrm{BW}_{\mathrm{ij}}(\mathrm{t})=\text { Bandwidth assigned for draining the queue } \\
& B W_{i j}(t) \eta_{i j}=\text { Queue depletion rate }
\end{aligned}
$$

The initial state of the queue $Q_{i j}(0)$ represents the initial size of the backlogged data of the queue. There is a possibility that IR ( $t$ ) may get fluctuated over time depending on the source behavior and can be viewed as the disturbance to the system. In general it is denoted using Eq. 2:

$$
\operatorname{IR}(t)=I_{n}+\omega(t)
$$

where, $I_{n}$ is a normal value of the input rate and $\omega(t)$ is a disturbance which can be either stochastic (e.g., white noise Gaussian process) or deterministic (e.g., impulse traffic load).

This disturbance can occur due to the randomness of the packet arrival from the applications (Kun et al., 2011).

\subsection{Estimation of Channel Condition}

The physical layer constraints such as channel fading, multi-path propagation, reflection, scattering and other climatic effects on the channel reveals the channel condition. This channel condition can be estimated based on the Received Signal Strength (RSS) and Signal to Noise Ratio (SNR) at the receiver.

The received signal strength (RSS) is estimated using Friis equation which is shown in Eq. 3:

$$
\mathrm{RSS}=\frac{\mathrm{P}_{\mathrm{tx}} * \alpha * \beta * \mathrm{H}_{\mathrm{tx}} * \mathrm{H}_{\mathrm{rx}} * \gamma^{2}}{(4 * \gamma * \mathrm{~d})^{2} * \delta}
$$

$$
\begin{aligned}
& \text { Where } \\
& \mathrm{P}_{\mathrm{tx}}=\text { Transmission power } \\
& \mathrm{A}=\text { Transmitter gain } \\
& \beta=\text { Receiver gain } \\
& \mathrm{H}_{\mathrm{tx}}=\text { Height of the transmitter } \\
& \mathrm{H}_{\mathrm{rx}}=\text { Height of the receiver } \\
& \gamma=\text { Wavelength } \\
& \mathrm{D}=\text { Distance between the transmitter and receiver } \\
& \delta=\text { System loss }
\end{aligned}
$$

From the above computed RSS, the Signal To Noise Ratio (SNR) is computed using Eq (4) (Jagadeesh and Purusothaman, 2011):

$$
\mathrm{SNR}=\log \left(\mathrm{P}_{\mathrm{tx}}\right)-\log \left(\mathrm{P}_{\mathrm{rx}}\right) \mathrm{dB}
$$

\subsection{Proposed Work}

Our proposed scheme operates in the Base Station (BS). BS stores the following parameters at different time intervals:

- Maximum available Buffer $\left(\mathrm{B}_{\mathrm{av}}\right)$

- Total Buffer under execution process $\left(\mathrm{B}_{\mathrm{e}}\right)$

- Number of applications accessing base station(NA)

- Queue length (Q)

- Flow Rate(R)

- Receive Signal Strength (RSS)

BS updates the above parameters periodically as per the application requirements. When a Buffer Request Packet (BRP) arrives from a specific application, the request packet is either allocated with available buffer or dropped. This is demonstrated as follows.

\subsection{Fuzzy Based Buffer Allocation}

Upon receiving BRP, the buffers are allocated using Buffer Allocation Factor (BAF). It is estimated with the help of fuzzy controller (Fig. 1). The steps involved in the fuzzy logic technique are detailed below:

- Fuzzification

- Inference with rule base

- Defuzzification

\subsection{Fuzzification}

In this step, the crisp inputs are changed into linguistic values. Each of these values is represented 
using a fuzzy set. Each fuzzy set is related to a membership function which is utilized to describe the way by which the crisp input belongs to the set.

Our technique considers four input parameters for Fuzzification such as number of User Requests (UR), flow Rate (R), Queue length (Q) and Received Signal Strength (RSS). Based on the input parameters and inference engine, the output obtained is the Buffer Allocation Factor (BAF). Each of the fuzzy parameters is represented using triangular membership function as it represents minimum and maximum and maximum boundary conditions. The membership to each of fuzzy variables is assigned using intuition method. This technique minimizes the computation complexity.

The membership function for these input parameters and output is represented as $f(U R), f(R), f(Q), f(R S S)$ and $\mathrm{f}$ (BAF) (Fig. 2-6).

\subsection{Number of User Requests}

Based on the count of user request, linguistic values associated with the membership function $f(U R)$ are low and high. The low UR is preferred for buffer allocation.

\subsection{Flow Rate}

The flow rate varies based on the user requirements as per the applications. They considered since they provide the required buffers. The variation level in the rate of flow is represented by using linguistic values related to the membership function $f(R)$ such as low and high. The high $\mathrm{R}$ is preferred for buffer allocation.

\subsection{Queue Length}

The queue length is measured based on the number of tasks in each queue i.e., it gives the measure for buffer availability. The linguistic values associated with the membership function $f(Q)$ are low and high. The higher $\mathrm{Q}$ is preferred for buffer allocation.

\subsection{Received Signal Strength}

The received signal strength describes the communication quality among the two nodes. The linguistic values associated with the membership function $\mathrm{f}$ (RSS) are low and high. The higher RSS is preferred for buffer allocation.

\subsection{Buffer Allocation Factor}

Output of four input linguistic value is buffer allocation factor. The allocation factor is represented by linguistic values associated with membership values such as low, medium and high.

The fuzzy buffer allocation scheme forms a fuzzy set of dimension $\mathrm{f}(\mathrm{UR}) * \mathrm{f}(\mathrm{R}) * \mathrm{f}(\mathrm{Q}) * \mathrm{f}(\mathrm{RSS})$.

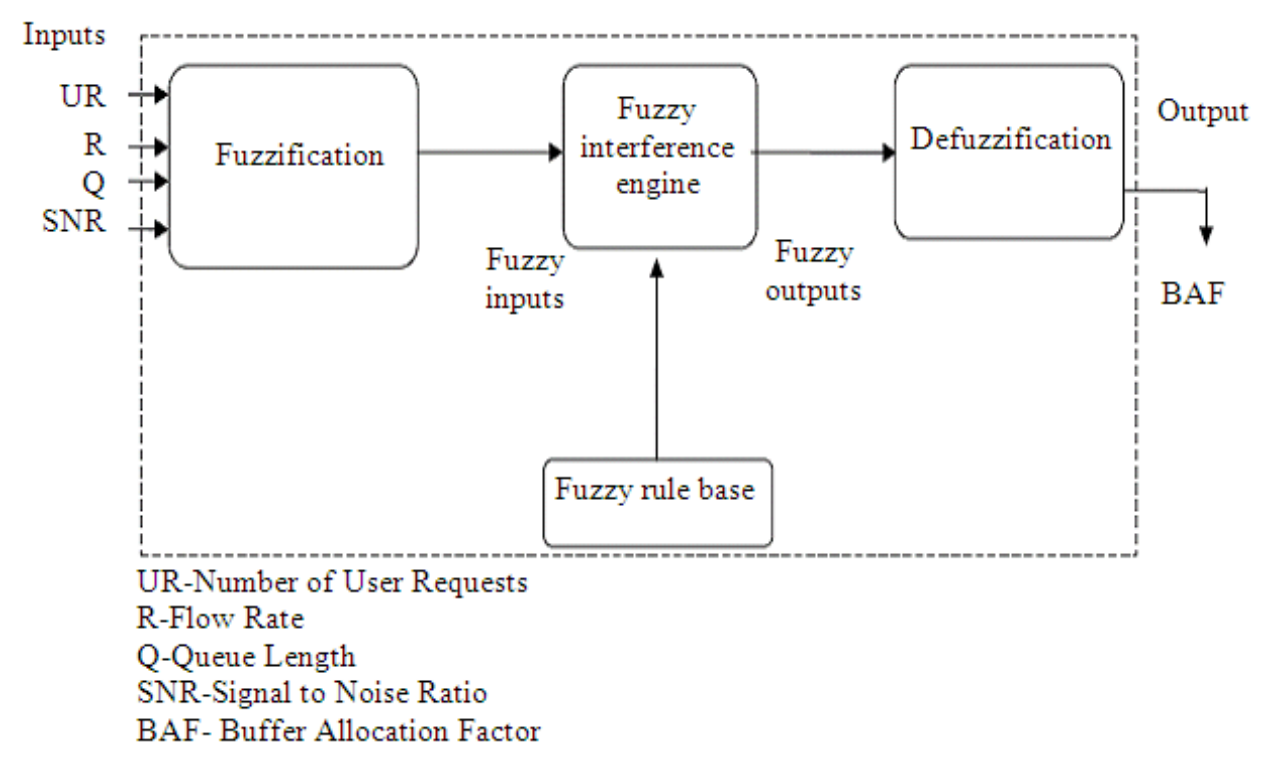

Fig. 1. Fuzzy controller for buffer allocation 
Karunkuzhali, D. and Dinesh Chandra Tomar / Journal of Computer Science 8 (12) (2012) 1946-1956

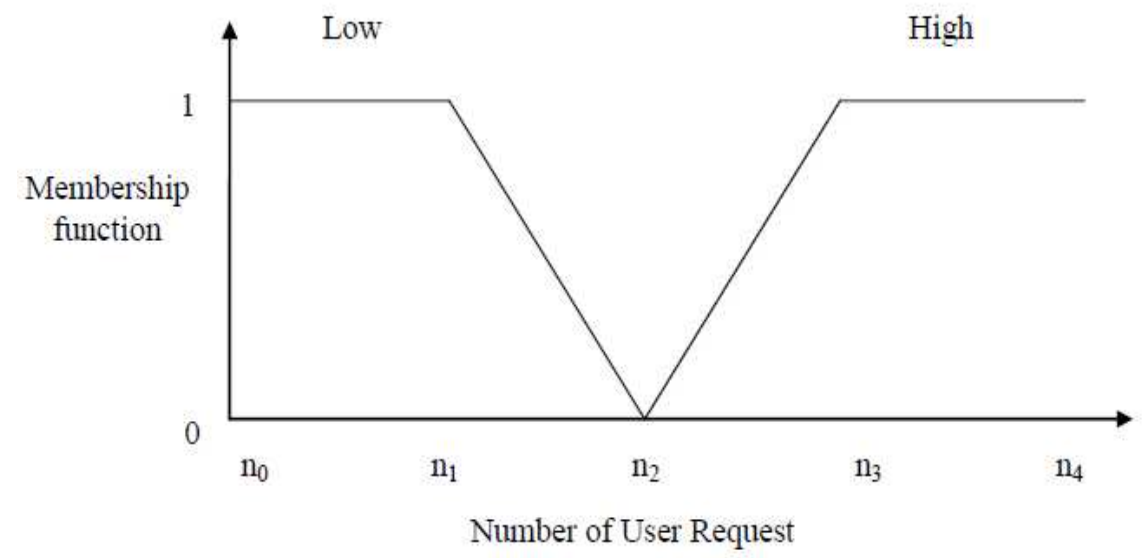

Fig. 2. Membership function for User Request

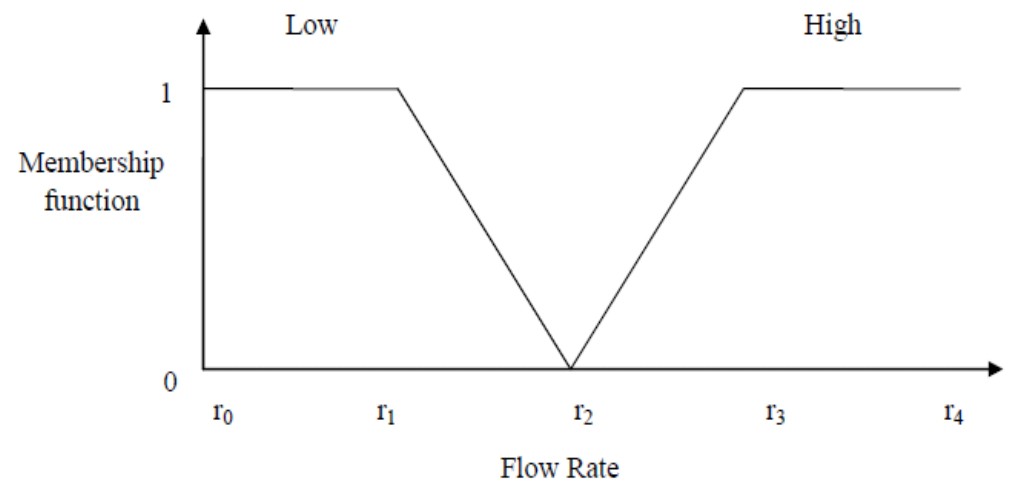

Fig. 3. Membership function for Flow Rate

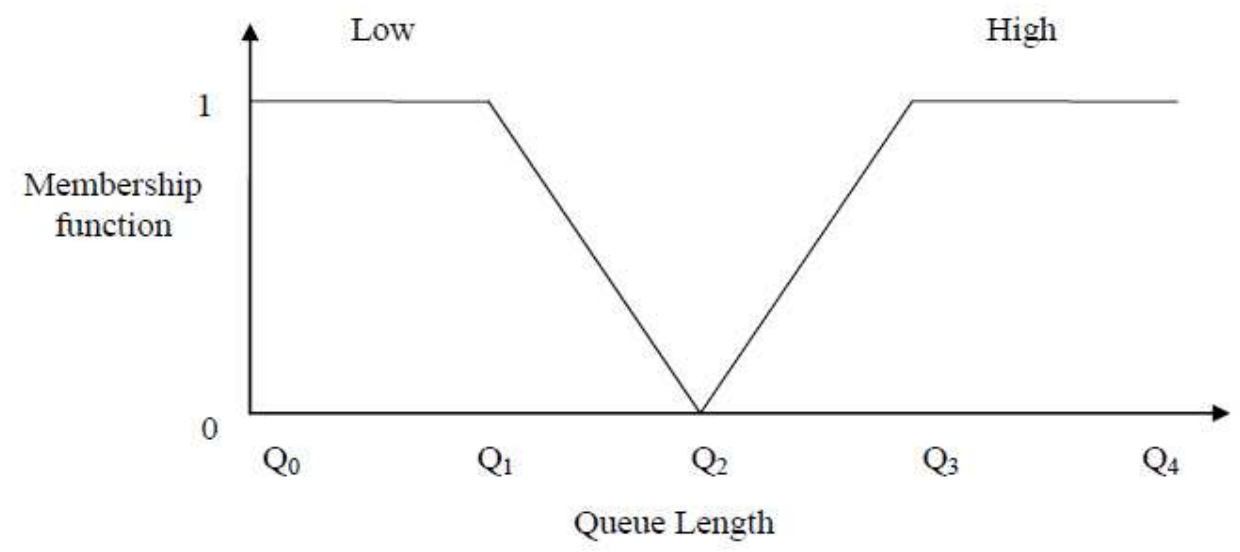

Fig. 4. Membership function for queue length 


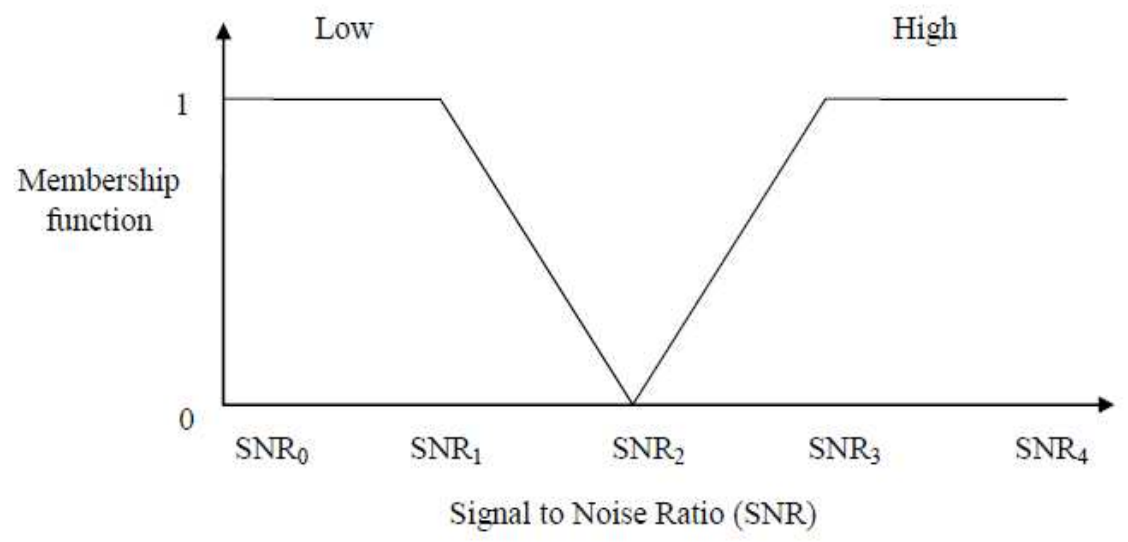

Fig. 5. Membership function for signal to noise ratio

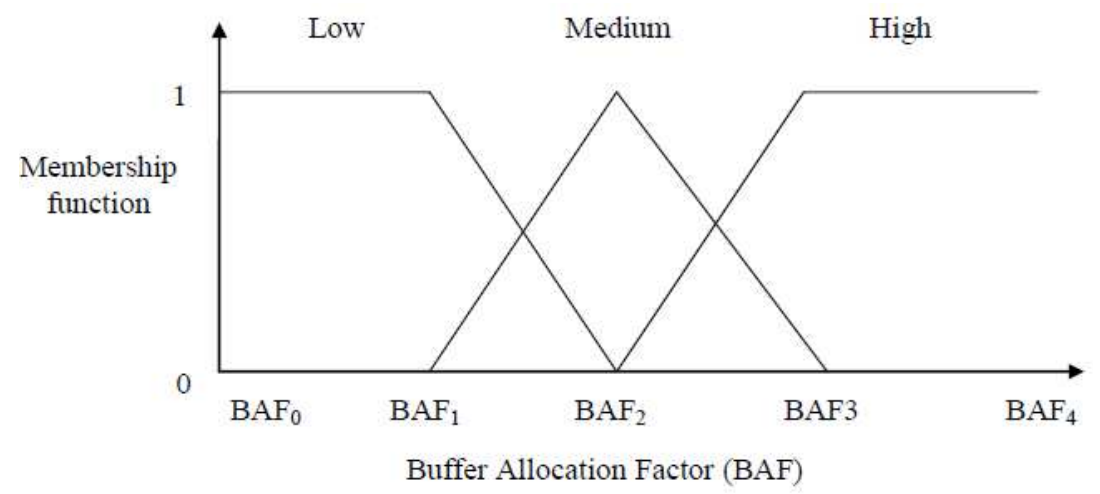

Fig. 6. Membership function for buffer allocation factor

Table 1. Fuzzy rules

\begin{tabular}{lllll}
\hline User requests & Flow rate & Queue length & RSS & BAF \\
\hline Low & Low & Low & Low & Low \\
Low & Low & Low & High & Medium \\
Low & Low & High & Low & Medium \\
Low & Low & High & High & High \\
Low & High & Low & Low & Medium \\
Low & High & Low & High & Medium \\
Low & High & High & Low & High \\
Low & High & High & High & High \\
High & Low & Low & Low & Low \\
High & Low & Low & High & Low \\
High & Low & High & Low & Low \\
High & Low & High & High & Medium \\
High & High & Low & Low & Low \\
High & High & Low & High & Medium \\
High & High & High & Low & Medium \\
High & High & High & High & High \\
\hline
\end{tabular}

\subsection{Inference Mechanism}

Inference mechanism in fuzzy logic is based on fuzzy rules that connect input and output parameters (fuzzy rule base) and the membership functions for input and output parameters. To create an inference engine, first the membership functions for input and output parameters are developed; both a range of values and a degree of membership define membership functions.

The fuzzy inference system is designed based on 16 rules described in Table 1. In order to demonstrate the designed fuzzy inference system, one rule is taken into account to show how the inference engine works and outputs of each rule are each rule are combined for generating fuzzy decision.

Rule 1

If $(\mathrm{UR}=$ low, $\mathrm{R}=$ high, $\mathrm{Q}=$ high and $\mathrm{RSS}=$ high $)$ Then 
$\mathrm{BAF}=$ high

End if

\subsection{Defuzzification}

It is the method by which a crisp value is extracted from a fuzzy set as illustration value. During fuzzy decision making, the centroid of area technique is taken into account for defuzzification. The Defuzzifier is based on Eq. 5:

$$
\left.\mathrm{F}_{-} \text {priority }=\sum_{\text {All_rules }} \mathrm{z}_{\mathrm{i}}^{*} \eta\left(\mathrm{z}_{\mathrm{i}}\right)\right] /\left[\sum_{\text {all_rules }} \eta\left(\mathrm{z}_{\mathrm{i}}\right)\right.
$$

Where:

$$
\begin{array}{ll}
\mathrm{F} \_ \text {priority } & =\text { Degree of decision making } \\
\mathrm{z}_{\mathrm{i}} & =\text { Fuzzy variable } \\
\eta\left(\mathrm{z}_{\mathrm{i}}\right) & =\text { Membership function }
\end{array}
$$

The output of the fuzzy priority function is altered to the crisp value based on the above defuzzification method.

Algorithm for Buffer Allocation

\section{Step 1}

BS estimates the parameters such as flow rate, queue length, number of user requests and received signal strength and updates the values periodically

Step 2

When the buffer request packet arrives, BAF is estimated as per step 3 .

Step 3

BAF estimation involves fuzzy logic technique which takes the estimated parameters in step 1 as input and BAF values are obtained as output.

Step 4

The user requests are sorted in the descending order of BAF

$$
\text { i.e., If } \mathrm{BAF}=\text { high }
$$

Then

$$
\text { User*BAF) }
$$

Allocate the Buffer

Buffer Allocated $=$ (Buffer Requested by queue.

Remaining flow requests waits in a pending

End if

\section{Packet Dropping}

After buffer allocation, when a new request arrives, the following steps are executed.

Step 1

When a new request arrives, its BAF is estimated.

If $\mathrm{BAF}=$ low
Then

The request is rejected.

Else

Goto step 2

End if

Step 2

The flow requests $\left(\mathrm{F}_{\mathrm{i}}\right)$ in the pending queue is verified for the channel condition.

If $\operatorname{RSS}\left(\mathrm{F}_{\mathrm{i}}\right)=$ low

Then

$F_{i}$ is dropped.

Buffer allocated for new request.

Else

End if

New request waits in the queue.

\section{RESULTS AND DISCUSSION}

\subsection{Simulation Model and Parameters}

Network Simulator (NS2) is used evaluate performance of the proposed Fuzzy Based Dynamic Buffer Management (FBDBM) scheme. The proposed scheme is implemented over IEEE 802.16 MAC protocol. In the Simulation, clients (SS) and the Base Station (BS) are deployed in a $1000 \times 1000 \mathrm{~m}$ region for $50 \mathrm{sec}$ simulation time. All nodes have the same transmission range of $500 \mathrm{~m}$. In the simulation, the CBR traffic is used. There are 8 downlink traffic flows from BS to SS.

The simulation settings and parameters are summarized in Table 2.

\subsection{Performance Metrics}

We compare our proposed FBDBM scheme with the MWRR (Mardini and Alfoul, 2011) scheme. We mainly evaluate the performance according to the following metrics.

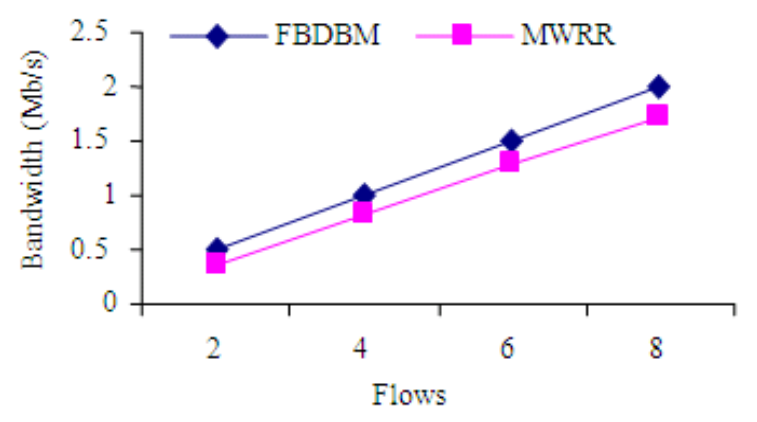

Fig. 7. Flow Vs received bandwidth 


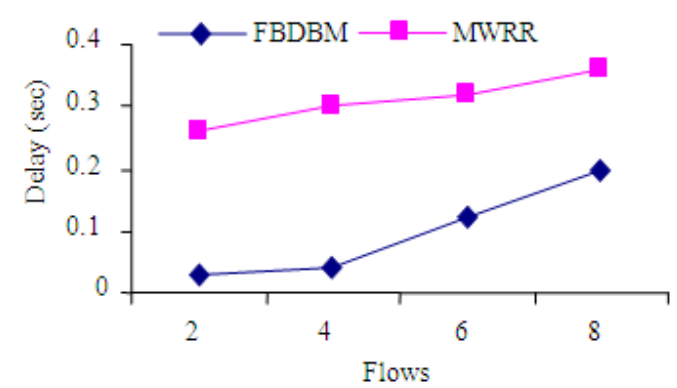

Fig. 8. Flow Vs delay

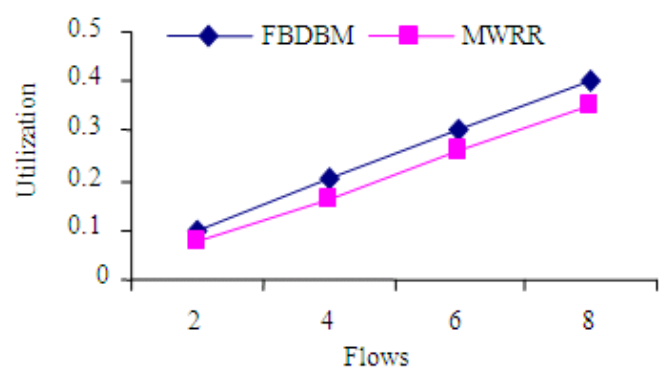

Fig. 9. Flow Vs utilization

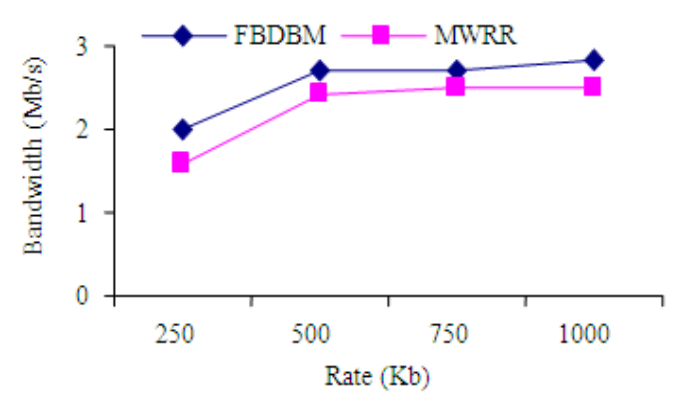

Fig. 10. Rate Vs received bandwidth

\subsection{Aggregated Bandwidth}

We measure the received bandwidth (in $\mathrm{Mb} / \mathrm{s}$ ) for CBR traffic of all flows

\subsection{Bandwidth Utilization}

For each flow, we measure the utilization as the ratio of bandwidth received of each flow to the available channel bandwidth.

\subsection{Average End-to-End Delay}

The end-to-end-delay is averaged over all surviving data packets from the sources to the destinations.
The performance results are presented as follows.

\subsection{Results}

\subsubsection{Effect of Varying the Traffic Flows}

In order to measure the impact of buffer allocation on the traffic flows, we vary the CBR downlink traffic flows from 2 to 8 .

When the number of traffic flows is increased, naturally the received bandwidth should also increase gradually, as we can see from the Fig. 7. It gives the aggregated received bandwidth for various traffic flows. From the figure, it can be seen that FBDBM has received slightly more bandwidth when compared to MWRR scheme, since buffer space is allocated based on traffic rate and queue size for each SS.

Figure 8 gives the average end-to-end delay for various traffic flows. From the figure, we can see that the average end-to-end delay of the proposed FBDBM scheme is less when compared to the MWRR scheme.

Since FBDBM allocates the buffer based on the number of user requests, it has better utilization. Figure 9 gives the bandwidth utilization for different traffic flows. From the figure, it can be seen that FBDBM achieves better utilization when compared with MWRR scheme, while increasing the traffic flows.

\subsection{Effect of Varying Transmission Rates}

In the first experiment, we vary the CBR traffic rate as $250,500,750$ and $1000 \mathrm{~Kb}$ for the 6 flows. The results are given as follows.

When the traffic rate is increased, naturally the received bandwidth should also increase, as we can see from the Fig. 10. It shows the aggregated received bandwidth for increased CBR traffic rates.

From the figure, it can be seen that FBDBM has received slightly more bandwidth when compared with MWRR scheme, since buffer space is allocated based on traffic rate and queue size for each SS.

Figure 11 gives the average end-to-end delay for various rate values. From the figure, we can see that the average end-to-end delay of the proposed FBDBM scheme is slightly less when compared to MWRR scheme.

Since FBDBM allocates the buffer based on the number of user requests, it has better utilization. Figure 12 give the bandwidth utilization for different traffic flows. From the figure, it can be seen that FBDBM achieves better utilization when compared with MWRR scheme, while increasing the traffic rate. 


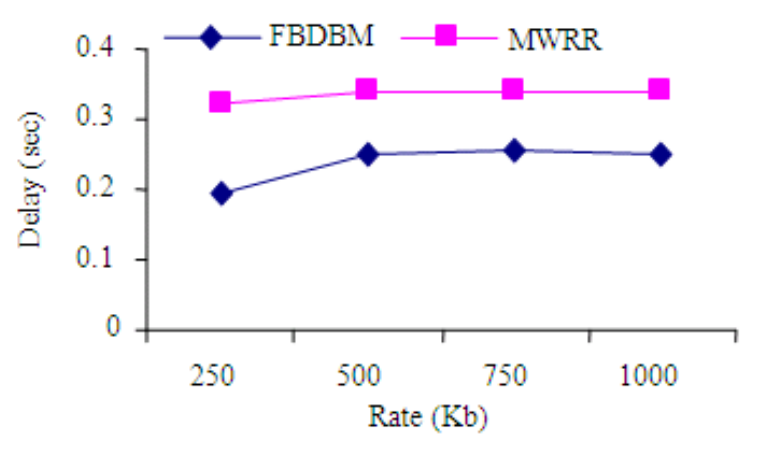

Fig. 11. Rate Vs delay

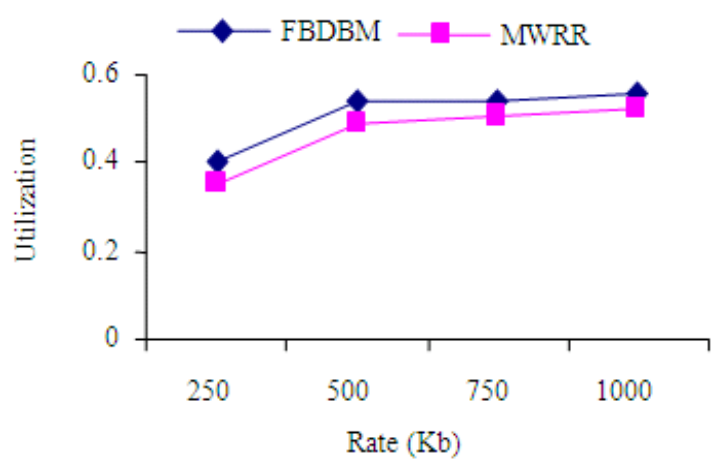

Fig. 12. Rate Vs utilization

Table 2. Simulation settings

\begin{tabular}{ll}
\hline Area size & $1000 \times 1000$ \\
\hline Mac & 802.16 \\
Clients & 10 \\
Radio range & $500 \mathrm{~m}$ \\
Simulation time & $50 \mathrm{sec}$ \\
Routing protocol & DSDV \\
Traffic source & CBR \\
Physical layer & OFDM \\
Channel error rate & 0.01 \\
Packet size & 1500 bytes \\
Frame duration & 0.005 \\
Transmission rate & $250 \mathrm{~Kb}, 500 \mathrm{~Kb}, 750 \mathrm{~Kb}, 1000 \mathrm{~Kb}$ \\
No. of flows & $2,4,6,8$ \\
\hline
\end{tabular}

\section{CONCLUSION}

In this study, we have proposed a fuzzy based dynamic buffer management in WiMAX 16 m network. The Base Station (BS) estimates the parameters such as number of user requests, flow rate, queue length and received signal strength and updates periodically. When a buffer request packet arrives at $\mathrm{BS}$, buffer allocation factor is estimated using the fuzzy logic applied over the parameters estimated in BS. The user requests are sorted in the descending order of BAF. This reveals that the flow request with more BAF is admitted and rest of the flow requests await in queue. When a new request arrives, its BAF is tested. If the value is low, the request packet is dropped. Otherwise, the pending request packet in the queue is emptied on analyzing their channel condition and buffer is allocated for new request. By simulation results, we have shown that the proposed technique reduces packet losses.

\section{REFERENCES}

1. Jagadeesh, S.J.K. and T. Purusothaman, 2011. An enhanced scheduling scheme for QoS guarantee using channel state information in WiMAX networks. Eur. J. Sci. Res., 64: 285292.

http://www.europeanjournalofscientificresearch. com/ISSUES/EJSR_64_2_10.pdf

2. Jin, B.L., 2010. Cooperative resource management in cognitive WiMAX with femto cells. Proceedings of the 29th Conference on Information, (CI' 10), IEEE Press Piscataway, N.J., USA., pp: 552-560. http://dl.acm.org/citation.cfm?id=1833623

3. Kim, S.J., B.B. Lee, S.W. Ryu, H.W. Lee and C.H. Cho, 2012. Cost effective coverage extension in IEEE802.16j based mobile WiMAX systems. Quality Service Resource Allocation WiMAX" InTech.

4. Kun, Z., D. Niyato and P. Wang, 2011. Dynamic service selection and bandwidth allocation in ieee $802.16 \mathrm{~m}$ mobile relay networks. IEEE J. Selected Areas Commun., 30: $\quad 1798-1805$. http://www.bibsonomy.org/bibtex/28b0acc04bd b47ee76b5e86ed73037c60/dblp

5. Lakkakorpi, J., A. Karhula, J. Alanen, O.J. Moilanen and J. Moilanen, 2007. Active queue management for reducing downlink delays in WiMAX. Proceedings of the IEEE 66th Vehicular Technology Conference, Sept. 30Oct. 3, IEEE Xplore Press, Baltimore, MD., pp: 326-330. DOI: 10.1109/VETECF.2007.81

6. Lenas, S., S. Dimitriou, T. Tsapeli and V. Tsaoussidis, 2011. Queue-management architecture for delay tolerant networking. Proceedings of the 9th IFIP TC 6 International 
Conference on Wired/Wireless Internet Communications, (WWIC' 11), Springer-Verlag Berlin, Heidelberg, pp: 470-482. http://dl.acm.org/citation.cfm?id=2023141

7. Mallapur, J.D., S. Abidhusain, S.S. Vastrad and A.C. Katageri, 2010. Fuzzy based bandwidth management for wireless multimedia networks. Commun. Comput. Inform. Sci., 70: 81-90.

http://link.springer.com/chapter/10.1007/978-3642-12214-9_15

8. Mardini, W. and M.A. Alfoul, 2011. Modified WRR scheduling algorithm for WiMAX networks. J. Network Protocols Algorithms. http://macrothink.org/journal/index.php/npa/arti cle/view/751

9. Papapanagiotou, I., D. Toumpakaris, J. Lee and M. Devetsikiotis, 2009. A survey on next generation mobile wimax networks: Objectives, features and technical challenges. IEEE Commun. Surveys Tutorials., 11: 3-18. DOI: 10.1109/SURV.2009.090402

10. Thavasi, S. and N. Natarajan, 2011. An effective queue management scheme for data communication. J. Comput. Sci., 7: 348-351. DOI:10.3844/jcssp.2011.348.351

11. Riizwan, M. and M. Ibrahiim, 2011. Efficient architecture for QoS providence across WiMAX. Comput. Sci. Series, 10: 203-214. http://anale-

informatica.tibiscus.ro/download/lucrari/9-1-17Rizwan.pdf

12. Scalosub, G., P. Marbach and J. Liebeherr, 2010. Buffer management for aggregated streaming data with packet dependencies. Proceedings of the IEEE INFOCOM, Mar. 1419, IEEE Xplore Press, San Diego, CA., pp: 15. DOI: 10.1109/INFCOM.2010.5462235
13. So-In, C., R. Jain and A.K. Tamimi, 2009. Scheduling in IEEE 802.16e mobile WiMAX networks: Key issues and a survey. IEEE J. Selected Areas Commun., 27: 156-171. DOI: 10.1109/JSAC.2009.090207

14. Soni, G. and S. Kaushal, 2011. Mobile WiMAX technology and its comparison with various competing technologies. Int. J. Comput. Corporate Res., 1 : 1-24. http://www.ijccr.com/November2011/5.pdf

15. Xue, Y., H. Nguyen, K. Nahrstedt and V.N. Univ, 2007. CA-AQM: Channel-aware active queue management for wireless networks. Proceedings of the IEEE International Conference, Communications, Jun. 24-28, IEEE Xplore Press, Glasgow, pp: 4773-4778. DOI: 10.1109/ICC.2007.788 